\title{
Ecological and network analyses identify four microbial species with potential significance for the diagnosis/treatment of ulcerative colitis (UC)
}

Wendy $\mathrm{Li}^{1,2+}$, Yang Sun ${ }^{3 \dagger}$, Lin Dai ${ }^{4}$, Hongju Chen ${ }^{1,2,5}$, Bin Yi ${ }^{5}$, Junkun Niu ${ }^{3}$, Lan Wang ${ }^{3}$, Fengrui Zhang ${ }^{3}$, Juan Luo ${ }^{3}$, Kunhua Wang ${ }^{6}$, Rui Guo ${ }^{3}$, Lianwei Li ${ }^{1,2}$, Quan Zou' ${ }^{7}$ Zhanshan (Sam) Ma ${ }^{1,2,8^{*}}$ and Yinglei Miao ${ }^{3^{*}}$

\begin{abstract}
Background: Ulcerative colitis (UC) is one of the primary types of inflammatory bowel disease (IBD), the occurrence of which has been increasing worldwide. Although IBD is an intensively studied human microbiome-associated disease, research on Chinese populations remains relatively limited, particularly on the mucosal microbiome. The present study aimed to analyze the changes in the mucosal microbiome associated with UC from the perspectives of medical ecology and complex network analysis.

Results: In total, 56 mucosal microbiome samples were collected from 28 Chinese UC patients and their healthy family partners, followed by amplicon sequencing. Based on sequencing data, we analyzed species diversity, shared species, and inter-species interactions at the whole community, main phyla, and core/periphery species levels. We identified four opportunistic "pathogens" (i.e., Clostridium tertium, Odoribacter splanchnicus, Ruminococcus gnavus, and Flavonifractor plauti) with potential significance for the diagnosis and treatment of $U C$, which were inhibited in healthy individuals, but unrestricted in the UC patients. In addition, we also discovered in this study: (i) The positive-to-negative links (P/N) ratio, which measures the balance of species interactions or inhibition effects in microbiome networks, was significantly higher in UC patients, indicating loss of inhibition against potentially opportunistic "pathogens" associated with dysbiosis. (ii) Previous studies have reported conflicting evidence regarding species diversity and composition between UC patients and healthy controls. Here, significant differences were found at the major phylum and core/periphery scales, but not at the whole community level. Thus, we argue that the paradoxical results found in existing studies are due to the scale effect.

(Continued on next page)
\end{abstract}

\footnotetext{
*Correspondence: ma@vandals.uidaho.edu; miaoyinglei@yeah.net

${ }^{+}$Wendy Li and Yang Sun contributed equally to this work.

'Computational Biology and Medical Ecology Lab, State Key Laboratory of

Genetic Resources and Evolution, Kunming Institute of Zoology, Chinese

Academy of Sciences, Kunming, China

${ }^{3}$ Department of Gastroenterology, The First Affiliated Hospital of Kunming

Medical University, Yunnan Institute of Digestive Disease, Kunming, Yunnan,

China

Full list of author information is available at the end of the article
}

C C The Author(s). 2021 Open Access This article is licensed under a Creative Commons Attribution 4.0 International License, which permits use, sharing, adaptation, distribution and reproduction in any medium or format, as long as you give appropriate credit to the original author(s) and the source, provide a link to the Creative Commons licence, and indicate if changes were made. The images or other third party material in this article are included in the article's Creative Commons. licence, unless indicated otherwise in a credit line to the material. If material is not included in the article's Creative Commons licence and your intended use is not permitted by statutory regulation or exceeds the permitted use, you will need to obtain permission directly from the copyright holder. To view a copy of this licence, visit http://creativecommons.org/licenses/by/4.0/ The Creative Commons Public Domain Dedication waiver (http://creativecommons.org/publicdomain/zero/1.0/) applies to the data made available in this article, unless otherwise stated in a credit line to the data. 
(Continued from previous page)

Conclusions: Our results reveal changes in the ecology and network structure of the gut mucosal microbiome that might be associated with UC, and these changes might provide potential therapeutic mechanisms of UC. The four opportunistic pathogens that were identified in the present study deserve further investigation in future studies.

Keywords: Inflammatory bowel disease, Ulcerative colitis, Mucosal microbiome, Species diversity, Species co-occurrence network, Core/periphery network

\section{Background}

Inflammatory bowel disease (IBD) exhibits chronic and relapsing inflammation of the gastrointestinal tract. Crohn's disease (CD), ulcerative colitis (UC), and ileal CD are the three most common phenotypes of IBD. The incidence of IBD is increasing worldwide, with over 3.5 million suffers in the United States and Europe [41]. However, the pathogenesis of IBD remains unclear, although may be related to the dysregulation of the internal mucosal environment due to changes in host genes, environmental factors, gut microbes, and immune responses [1, 28, 31, 35, 42, 45]. Studies have identified that variations in several genes are associated with IBD risk, including NOD2, ATG16L1, CARD9, and CLEC7A [28], with diet, medication, and geography also involved in disease development [1, 33].

The human gut microbiome plays a key role in nutrient metabolism, pathogen protection, and immune system development. Dysbiosis of the gut microbiome is also associated with IBD $[31,48]$. Common gut microbiome changes in IBD patients, for example, include a lower abundance of obligate anaerobic bacteria of short-chain fatty acids (SCFAs) and an increase in abundance of facultative anaerobes [15, 30, 42, $51,59]$. Through long-term continuous sampling of IBD patients and healthy controls, Halfvarson et al. [18] reported greater fluctuation in the gut microbiome of the IBD cohort than of healthy controls. Changes in the microbiome composition are often accompanied by gut function disorders [5, $16,22,35]$. For example, the species and microbial metabolites associated with oxidative stress responses are significantly increased in the gastrointestinal tract of IBD patients [16]. IBD may also affect secondary bile acid metabolism in the gut microbiome [22]. Moreover, core metabolic functions are persistent and redundant across multiple gut microbial phyla, despite temporal variations in microbial taxa, genomes, and proteomes [5]. Thus, the gut microbiome appears to play a key role in the pathogenesis of IBD, although the causal relationship between microbiome dysbiosis and IBD is still unclear. Therefore, correcting the gut microbiome or its functions in patients has become a target for IBD therapy, and can be achieved through various strategies, such as antibiotics, probiotics, and fecal microbial transplantation (FMT) [11, 32, 56, 63, 64].

The objective of the current study was to investigate the influence of UC on the intestinal mucosal microbiome from the perspective of medical ecology and complex network analysis. We analyzed 56 mucosal microbiomes from 28 Chinese UC patients and their healthy family partners from three aspects, including species diversity, shared species, and inter-species relationships. All analyses were performed at the whole community, main phylum, and core/periphery species network scales.

\section{Materials and methods Study design and sample collection}

All study procedures involving human subjects were approved by the Medical Ethics Board of the First People's Hospital of Yunnan Province, China. Written and verbal informed consent were obtained from all participants. Microbial samples of intestinal mucosa were collected from 28 couples. Each couple consisted of one UC patient and a healthy control. All 56 participants were from Kunming, China, and were between the ages of 18 and 60 years old. Healthy volunteers were free of gastrointestinal illnesses and did not use drugs during endoscopy, nor did they take antibiotics during the year prior to sample collection. The diagnosis of UC was based on standard endoscopic, radiographic, and histologic criteria. All patients had been under treatment with Mesalazine. Mucosal samples were collected in the morning from the participants without undergoing bowel cleansing preparation. The intestinal mucosal sample was taken $10 \mathrm{~cm}$ from the anus using disposable biopsy forceps. After sampling, mucosal samples were immediately frozen in liquid nitrogen and stored at $-80^{\circ} \mathrm{C}$ until DNA extraction.

\section{DNA extraction, 16S rRNA sequencing, and taxonomic assignment of reads}

Bacterial 16S rRNA genes were amplified by polymerase chain reaction (PCR) using barcoded primers flanking the hypervariable regions V3 and V4. Amplicons were sequenced using the Illumina pyrosequencer platform. Raw data were filtered to eliminate adapter pollution and low-quality reads to obtain high-quality clean reads. The overlapping paired-end reads were then merged to tags. In total, we obtained 3,062,675 tags without primers, with 27,345 tags per sample on average. Tags were clustered into operational taxonomic units (OTUs) using scripts in USEARCH (v7.0.1090) at 97\% sequence similarity [14]. OTU representative sequences were taxonomically classified using the Ribosomal Database 
Project (RDP) Classifier v.2.2 trained on the Greengenes database.

\section{Measuring microbiome diversity with Hill numbers}

Microbiome diversity was quantified using Hill [24] numbers, which were reintroduced to ecology by Jost [27] and Chao et al. [8, 9], defined as:

$$
q D=\left(\sum_{i=1}^{S} p_{i}^{q}\right)^{1 /(1-q)}
$$

where $D$ is the diversity, $q$ is the order number of diversity, $S$ is the number of species (or OTUs), and $p_{i}$ is the relative abundance of species $i$. Hill numbers at different $q$ orders correspond to special ecological diversity indices, in which ${ }^{O} D$ is equal to species richness, ${ }^{1} D$ represents the exponential of the Shannon index, and ${ }^{2} D$ represents the reciprocal of the Simpson index. The larger the diversity order $q$, the more sensitive ${ }^{q} D$ is to species with high abundance.

We used effect size calculated with Cohen's [10] $d$ statistic to examine differences in diversity between two groups. A $p$-value of $<0.05$ indicated significant difference in microbiome diversity between two groups.

\section{Shared species analysis}

The null hypothesis $\left(\mathrm{H}_{0}\right)$ of shared species (OTUs) is that the number of shared OTUs between the two groups is no less than that between any two random groups. The alternative hypothesis $\left(\mathrm{H}_{\mathrm{A}}\right)$ is that the number of shared OTUs between two groups is less than that between any two random groups. We applied two algorithms to estimate the number of shared OTUs between two random groups (expected number of shared OTUs). The first algorithm (A1) was applied to randomly reassign OTUs and samples in the two groups, as follows: (i) Total number of reads (abundances) for each OTU in the two groups was first computed. (ii) For each OTU, the reads from the two groups were pooled together. (iii) The number of reads of each OTU was randomly reassigned into two new groups, and the number of shared OTUs between these two new groups was computed. The total number of reads in each new group should remain the same as that in the corresponding observed group. (iv) Step (iii) was repeated 1000 times. (v) The pseudo $p$-value was finally calculated, as follows:

$$
p=D / 1000
$$

where $D$ is the number of times the numbers of expected shared OTUs from 1000 random reassignments exceeded the number of observed shared OTUs. A $p$ - value of $<0.05$ indicated strong evidence to reject the null hypothesis and accept the alternative hypothesis.

The second algorithm (A2) was used to randomly reassign samples only, with the following steps: (i) All samples from the two groups were pooled together. (ii) The samples were randomly reassigned into two new groups. The total number of samples in the two new groups should remain the same as that in the corresponding observed groups. The number of shared OTUs between the two new groups was computed. (iii) Step (ii) was repeated 1000 times. (iv) The pseudo $p$-value was calculated as follows:

$$
p=D / 1000
$$

where $D$ is the number of times that the number of expected shared OTUs from 1000 random reassignments exceeded the number of observed shared OTUs. A $p$ value of $<0.05$ indicated strong evidence to reject the null hypothesis and accept the alternative hypothesis.

\section{Species co-occurrence network ( $\mathrm{SCN}$ ) analysis}

To reduce the noise of spurious OTUs, we filtered those OTUs with total reads from all samples of $<25$. As the number of samples for each group was 28 , the OTUs removed were equivalent to singletons with approximately one read per sample. Spearman's correlation coefficients computed with the relative abundance of OTUs were adjusted with the false discovery rate (FDR) control with $p=0.05$. Cytoscape (v2.8.3) was used to visualize networks [54] and the iGraph R-package [12] was used to compute basic network properties. The MCODE plug-in [3] of Cytoscape was used to detect network clusters (modules). In addition, we also detected the positive-tonegative links $(\mathrm{P} / \mathrm{N})$ ratios in the SCNs, as introduced by Ma [36].

\section{Core/periphery network (CPN) analysis}

Core-periphery structures in a network consist of two classes of nodes, i.e., dense cohesive core nodes and sparse connected periphery nodes [6]. In an ideal coreperiphery network, core nodes are fully connected to each other and to some periphery nodes, whereas periphery nodes are not connected with other periphery nodes [13]. The objective function $(\rho)$ is used to measure how well the real structure approximates the ideal, defined as:

$$
\rho=\sum_{i, j} a_{i j} \delta_{i j}
$$

In the equation, $a_{i j}$ represents the presence or absence of the link between node $i$ and node $j$, where $a_{i j}=1$ if node $i$ and node $j$ are linked, and 0 otherwise. $\delta_{i j}$ indicates the presence or absence of a link between node $i$ 
and node $j$ in the ideal core/periphery network, where $\delta_{i j}=1$ if node $i$ or/and node $j$ are core, and 0 otherwise. Let $A$ be the adjacency matrix of $a_{i j}$, and $\Delta$ be the adjacency matrix of $\delta_{i j}$. When $A$ and $\Delta$ are identical, the measure $\rho$ achieves its maximum value. When $\rho$ is maximum, we can classify the node into either core or periphery based on $\delta$. We implemented the CPN analysis in Python using code provided by Ma \& Ellison [37].

\section{Results}

\section{Bioinformatics analysis of sequencing data}

The 28 UC patients included 11 females and 17 males, and 28 healthy controls included 17 females and 11 males. There were 842 and 860 OTUs in the healthy and UC groups, respectively. Fifteen known phyla were identified in the mucosal microbiome, with Firmicutes, Bacteroidetes, and Proteobacteria found to be dominant.

In addition, we built the SCNs based on the mucosal microbiomes of the healthy and UC subjects, and divided the OTUs (species) in each network into core and periphery groups using CPN analysis, which can reveal global characteristics of network structure and stability. The results are listed in Table S1. In the microbiome of healthy individuals, there were 146 core species and 170 periphery species. In the microbiome of UC patients, there were 190 core species and 206 periphery species.

\section{Mucosal microbiome diversity based on Hill numbers}

The alpha diversity of the mucosal microbiome was quantified using Hill numbers. For different diversity orders $(q)$, the Hill number corresponds to different ecological diversity indices, including species richness, Shannon, and Simpson indices. The influence of $\mathrm{UC}$ on the mucosal microbiome was explored at three levels, including the whole community, main phylum, and core/periphery species scales. Figure 1 and Table S2 display the diversity of the mucosal microbiomes based on Hill numbers at four diversity orders $(q)$ for healthy individuals and UC patients, as well as the significance test results.

First, the diversity of the whole mucosal microbiome was quantified with all species in the community sample. At the whole community level, there were no significant differences in the Hill numbers of the mucosal microbiomes between healthy individuals and UC patients (Table S2 and Fig. 1).

We next quantified the diversities of the three major phyla (i.e., Firmicutes, Bacteroidetes, and Proteobacteria) in the mucosal microbiomes, and determined the influence of UC. In general, the three most common phyla in the fecal microbiome followed the order Firmicutes $>$ Bacteroidetes $>$ Proteobacteria $[45,55]$. In the present study, Firmicutes had the highest Hill numbers in the mucosal microbiomes of both the healthy and UC




groups. In the mucosal microbiome of healthy individuals, the Bacteroidetes Hill number was significantly higher than that of Proteobacteria at the diversity order $q=0$ (Cohen's $d=0.82, p<0.05$ ). However, in the mucosal microbiome of UC patients, the Bacteroidetes Hill number was significantly lower than that of Proteobacteria at the diversity order $q=0$ (Cohen's $d=-0.73, p \leq$ 0.05 ). No significant differences were observed between the Bacteroidetes and Proteobacteria Hill numbers at the other three diversity orders $(q=1,2$, and 3$)$. At the diversity order $q=0$, the Firmicutes Hill number in the mucosal microbiome of healthy individuals was significantly higher than that of UC patients (Table S2). At the diversity orders $q=0$ and 1 , the Bacteroidetes Hill number in the mucosal microbiome of healthy individuals was significantly higher than that of UC patients. There was no significant difference in the Proteobacteria Hill number between healthy individuals and UC patients.

Third, we quantified the diversities of core and periphery species in the mucosal microbiome. At diversity orders $q=1,2$, and 3, the Hill numbers of the core species in the mucosal microbiome of the UC patients were significantly higher than that of the healthy individuals, although there was no significant difference in Hill numbers at $q=0$ between healthy and UC subjects (Fig. 1 and Table S2). There were no significant differences in the Hill numbers of periphery species between healthy individuals and UC patients. This indicated that UC mainly affected core species with high abundance.

\section{Shared species analysis}

Shared species analysis was used to detect differences in microbial composition between different groups. Table S3 lists the results of shared species analysis based on the two algorithms [38]. For each comparison, if the observed number of shared species was significantly smaller than that expected by chance, this indicated that the shared species between this group pair were not caused by chance, and there was a significant difference between their microbial compositions. We also performed shared species analysis of the mucosal microbiome at the three levels.

With the $A 1$ algorithm (reshuffling reads), the observed number of shared species between the group pairs was significantly smaller than that expected by chance for all comparisons (Fig. 2 and Table S3). Compared with the $A 1$ algorithm, the $A 2$ algorithm (reshuffling samples) was more conservative. In the whole mucosal microbiome, there was no significant difference in the observed number of shared species between the healthy and UC groups versus that expected by chance. For shared species analysis of the three main phyla, Firmicutes showed a similar result to that found at the whole community level. However, the observed number

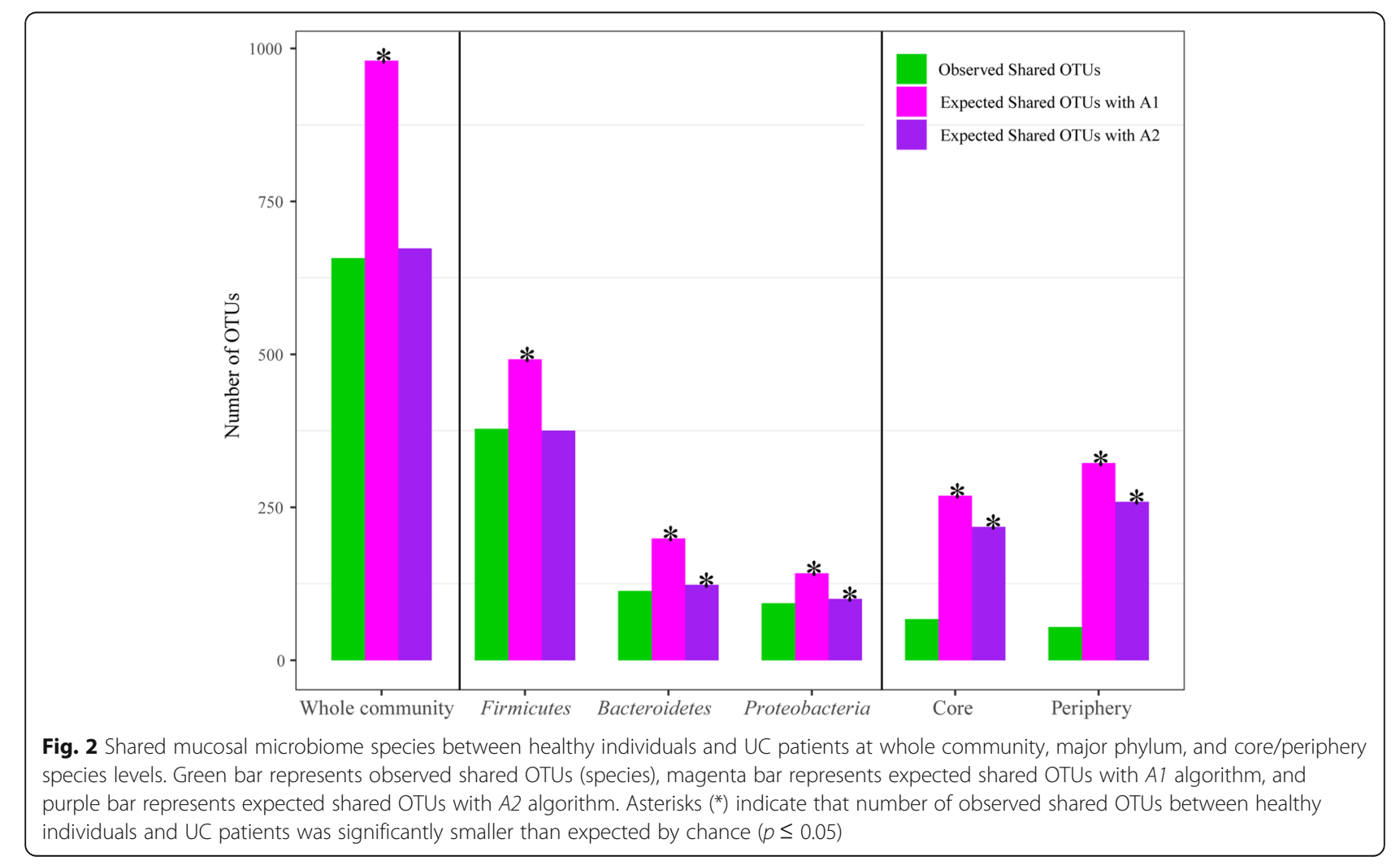


of shared Bacteroidetes and Proteobacteria species between healthy and UC subjects was significantly smaller than that expected by chance. Furthermore, the observed number of shared core/periphery OTUs was significantly smaller than that expected by chance. As shown in Table S4, the SCNs of the healthy and UC groups shared 67 core species and 54 periphery species. Table S5 shows the shared and specific core/periphery species of each group.

Based on the diversity and shared species results, UC appeared to have little effect on the mucosa microbiome at the whole community level. However, we found that UC was associated with dysbiosis of the mucosa microbiome, characterized by changes in the main bacterial phyla, including a decrease in Firmicutes richness, change in Proteobacteria species composition, and variation in both Bacteroidetes diversity and species composition. In addition, UC was also associated with changes in core and periphery species in the mucosal microbiome network. According to the Vellend-Hanson synthesis [21, 61], CPN analysis can effectively detect inequalities from a node perspective, which are caused by the selection effects of the mucosa or host environment $[34,39]$. Thus, the significant differences in the diversity and composition of the core/periphery species indicated that UC may influence the selection effects of the host environment on the OTUs in the mucosal microbiome.

\section{Species co-occurrence network ( $\mathrm{SCN}$ ) analysis}

We first removed relatively sparse OTUs from the two mucosal microbiomes (as described in the Materials and Methods). The mucosal microbiome $\mathrm{SCNs}$ of the healthy (healthy-SCN) and UC (UC-SCN) groups were then constructed, as illustrated in Figs. 3 and 4, respectively. Table $\mathrm{S} 6$ shows the basic network properties of the two SCNs. The number of nodes in the two SCNs was similar, but the number of edges in the UC-SCN was two times that in the healthy-SCN, which resulted in a higher average degree. The number of connected components in the healthy-SCN was nearly twice that of the UC-SCN, indicating that the fragmentation degree of the healthy-SCN was higher than that of the UC-SCN.

The MCODE algorithm was used to detect network clusters (modules). Results are shown in Table S7, and include the cluster number, cluster score, number of nodes, and number of edges for each cluster. The cluster score is a measure of cluster density, with a higher cluster score indicating a stronger corresponding cluster. The healthy-SCN had only one strong cluster (\#1 cluster, score $>5$ ), whereas the UC-SCN had two strong clusters (\#1 and \#2 clusters, scores > 5). These three clusters consisted of species from the phyla Firmicutes and Proteobacteria. As shown in Fig. 3b, 85\% (22/26) of OTUs (nodes) in the \#1 cluster of the healthy-SCN belonged to Firmicutes and Proteobacteria. As shown in Fig. $4 \mathrm{~b}$ and c, in the UC-SCN, 75\% (18/24) of OTUs in the \#1 cluster and all OTUs in the \#2 cluster were from Firmicutes and Proteobacteria. Bacteroidetes species were scattered in both SCNs without forming a strong cluster. The healthy-SCN \#1 cluster and UC-SCN \#2 cluster had similar species composition and network structure. For species composition, 15 OTUs were shared between the two clusters (see Table S8). For network structure, all interactions within the two clusters were positive/cooperative, and these clusters both contained more than half of the negative/inhibitive interactions of their SCNs (Figures S1 and S2). Thus, these two clusters may play a similar role in their own mucosal microbiome.

In addition, based on the $\mathrm{P} / \mathrm{N}$ ratios in the $\mathrm{SCNs}$, $\mathrm{UC}$ was associated with a loss in mucosal microbiome balance in patients. As shown in Table $\mathrm{S} 9$, the $\mathrm{P} / \mathrm{N}$ ratio in the UC-SCN was five times higher than that in the healthy-SCN, indicating that the number of inhibitive interactions decreased in the mucosa microbiome of UC patients. The negative links were mainly found within the three main phyla, i.e., Firmicutes, Bacteroidetes, and Proteobacteria. In the healthy-SCN, 59\% (45/76) of negative links were found within Firmicutes and 22\% (17/76) were found between Firmicutes and Proteobacteria. In the UC-SCN, the number of negative links decreased to less than half that in the healthy-SCN, which was mainly due to the considerable reduction of negative links within Firmicutes and between Firmicutes and Bacteroidetes. Compared with the healthy-SCN, we identified a marked increase in the $\mathrm{P} / \mathrm{N}$ ratios within and among the three main phyla in the UC-SCN. As mentioned above, we used CPN analysis to divide the OTUs in the network into two groups, i.e., core and periphery. In both the healthy- and UC-SCNs, more than $50 \%$ of positive and negative links were found within the core species. Similar to the patterns observed at the whole community and main phylum scales, the core, periphery, and coreperiphery species in the UC-SCN had higher $\mathrm{P} / \mathrm{N}$ ratios than those in the healthy-SCN.

\section{Potential "pathogens" or research targets of UC}

The five times higher $\mathrm{P} / \mathrm{N}$ ratio in $\mathrm{UC}$ patients signaled loss of inhibition to certain potentially opportunistic pathogens. Based on the changes in abundance and $\mathrm{P} / \mathrm{N}$ ratio of each OTU in UC patients, we set the following selection criteria for potential pathogens: $(i)$ significant differences in abundance between the healthy and UC groups (Wilcoxon test $p \leq 0.05$ ); (ii) five or more negative links and a $\mathrm{P} / \mathrm{N}$ ratio of $<0.5$ in the healthy-SCN; but (iii) a significantly higher $\mathrm{P} / \mathrm{N}$ ratio in the UC-SCN.

In total, four OTUs or species met the selection criteria, including Clostridium tertium (OTU ID: 175), 


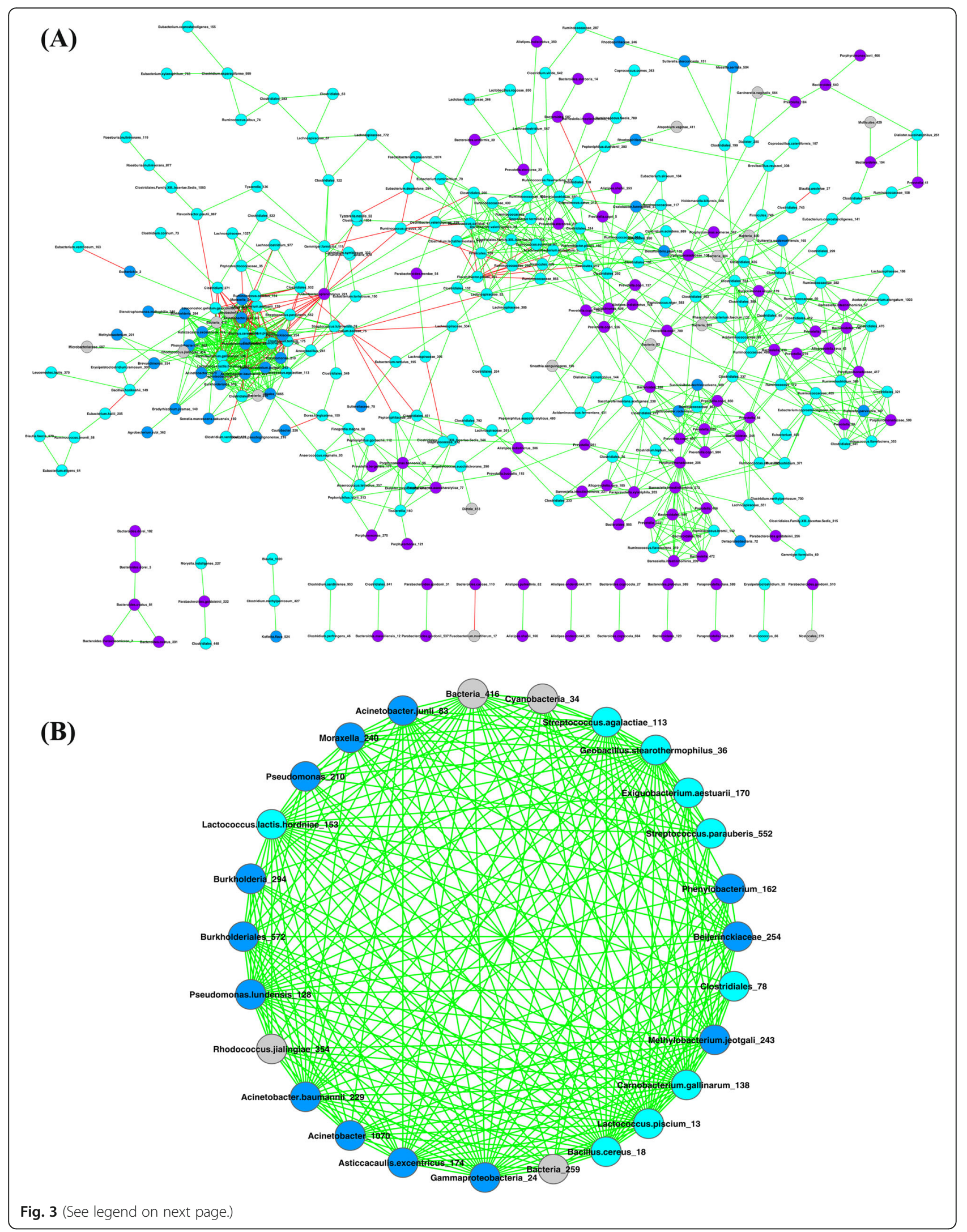


(See figure on previous page.)

Fig. 3 Mucosa microbiome SCN of healthy group and strongest cluster (score > 5): a Mucosa microbiome SCN of healthy group; b Strongest cluster (\#1 cluster). Nodes in cyan, OTUs of Firmicutes phylum; nodes in blue, OTUs of Proteobacteria phylum; nodes in purple, OTUs of Bacteroidetes phylum; nodes in gray, OTUs of other phyla; edges in green, positive correlations; edges in red, negative correlations

Odoribacter splanchnicus (OTU ID: 221), Ruminococcus gnavus (OTU ID: 32), and Flavonifractor plautii (OTU ID: 161) (see Table S10 for details). Results showed an increase in C. tertium, $R$. gnavus, and F. plautii, but a decrease in O. splanchnicus in the mucosal microbiome of UC patients. In addition, $C$. tertium had the greatest number of negative links in the healthy individuals, but only one negative link in the UC patients. Both $O$. splanchnicus and F. plautii had 11 and nine negative links, respectively, in healthy individuals, but none in UC patients. In the healthy-SCN, $R$. gnavus only had negative links (no positive links), whereas, in the UC$\mathrm{SCN}, R$. gnavus had 1.5 times more positive links than negative links. Furthermore, C. tertium and O. splanchnicus were core species in both healthy and UC subjects, whereas, $R$. gnavus and F. plautii were core species in healthy individuals but periphery species in UC subjects.

\section{Discussion and conclusions}

We compared mucosal microbiome samples from 28 UC patients and their healthy partners. Analyses focused on species diversity, shared species, and species interactions at three scales (i.e., whole community, major phylum, and core/periphery critical network). Our study revealed three major findings, as summarized below.

First, the differences between UC patients and healthy controls in diversity and shared species were scale dependent. At the whole community scale, the differences in species diversity and composition were not obvious. However, at the major phylum and core/ periphery species scales, significant differences were found between the UC patients and healthy controls. Although a reduction in gut microbial diversity in UC patients has been reported in multiple studies [31, 43, $46,65]$, other research has found there to be no significant differences between UC patients and healthy individuals $[7,20,52]$. These inconsistencies are likely related to differences in study design, subject status, and sample collection processes. Compared with the whole gut mucosal microbiome, compositional alterations at the phylum level are more consistent across different studies, e.g., decrease in Firmicutes and Bacteroidetes [31, 45] and increase in Proteobacteria [16, 44]. Moreover, the compositional variation at taxonomic scales plays an important role in explaining pathogenesis and assessing therapeutic efficacy. For example, Ishikawa et al. [25, 26] found that FMT following antibiotic pretreatment can effectively improve gut dysbiosis caused by loss of Bacteroidetes in UC patients. The significance of our first finding reinforces the critical importance of scale in studies on microbiome-associated diseases, and provides the core/periphery network structure as a new scale for microbiome research.

Second, it is well recognized that UC is associated with dysbiosis or loss of balance in the gut microbiome of patients $[11,31,44,46,51]$. However, no existing studies have offered quantitative measures to demonstrate the effects of dysbiosis. In other words, the characterization of dysbiosis is often presented in the form of qualitative description. Our study revealed a considerably higher (five times) $\mathrm{P} / \mathrm{N}$ ratio in UC patients, signaling the loss of inhibition to certain potentially opportunistic pathogens. It should be noted that we use the term "pathogen" somewhat differently from the traditional Koch's postulates, i.e., certain bacterial species are responsible for disease. Current research on microbiome-associated diseases still cannot, in many cases, answer the simple question-what are the disease pathogens based on the traditional Koch's postulates?

Third, the considerable change in $\mathrm{P} / \mathrm{N}$ ratio not only quantified the effects of dysbiosis but also helped to detect the "culprits". Based on the variations in composition and $\mathrm{P} / \mathrm{N}$ ratio, we identified four potentially opportunistic pathogens, i.e., C. tertium, $O$. splanchnicus, R. gnavus, and F. plautii. Clostridium tertium is an anaerobic gram-positive bacterium in the phylum Firmicutes. Although $C$. tertium has traditionally been considered non-pathogenic, various studies have reported it to be a significant cause of bacteremia and other infections in neutropenia patients $[29,49,50,53,60]$. In addition, C. tertium infections in non-neutropenic patients have also been reported recently $[57,58,62]$. Odoribacter splanchnicus (Bacteroides splanchnicus) is an anaerobic gramnegative bacterium in Bacteroidetes. Although it is found in normal human colonic microbiomes, it has the potential to be an opportunistic pathogen [17]. In the present study, we found reduced abundance of $O$. splanchnicus in UC patients, consistent with that reported in CD patients [42]. Odoribacter splanchnicus can produce acetate, propionate, and butyrate, which are associated with the biosynthesis of SCFAs [17]. 


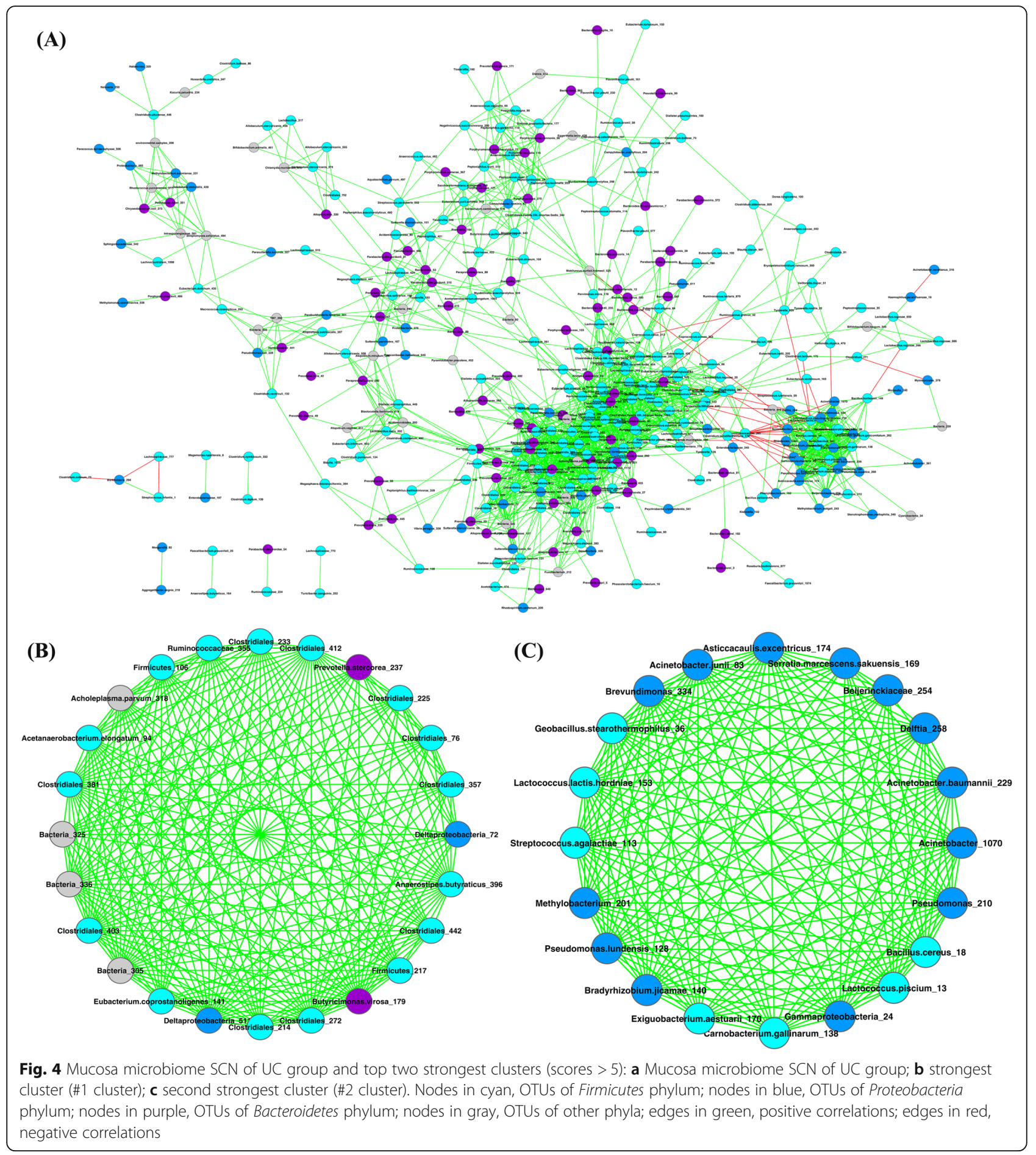

The effect of $O$. splanchnicus variation on UC patients may also be related to the reduction in SCFAs $[17,42]$. Ruminococcus gnavus is an anaerobic grampositive bacterium in the phylum Firmicutes. An increase in $R$. gnavus has been reported previously in the gut microbiome of IBD patients [19, 46, 65], as found in the present study. Henke et al. [23] recently reported that $R$. gnavus can produce an inflammatory polysaccharide, revealing a possible relationship between $R$. gnavus and IBD pathogenesis. Flavonifractor (Eubacterium) plautii is another anaerobic grampositive member of Firmicutes. Compared with 
healthy individuals, patients with IBD have significantly higher concentrations of mucosal IgG in their gut, although corresponding antigens remain unclear $[4,40]$. Recent studies have found that $F$. plautii is associated with enhancement of host intestinal IgG levels in IBD patients $[2,47]$. In summary, our findings reinforce the potential importance of these four species, which deserve further investigation in future studies.

\section{Supplementary Information}

The online version contains supplementary material available at https://doi. org/10.1186/s12866-021-02201-6.

\section{Additional file 1.}

\section{Acknowledgements}

Not applicable.

\section{Authors' contributions}

Y.S., J.N., and W.L. collected the human specimens and data. W.L. performed data analysis and drafted the manuscript. L.D., H.C., B.Y., F.Z., K.W., J.L., R.G., L.L. \& Q.Z. participated in the discussion and interpretations of the results; W.L., Y.S., Z.S.M. \&Y.L.M. designed the study and revised the manuscript. All authors approved the submission.

\section{Funding}

This work was supported by National Natural Science Foundation of China (U1802282, 82060107, 81660100, 81670501), Applied Basic Research Projects of Yunnan Province, China [202001AY070001-202], Open Grants (\#GREKF1814, \#GREKF19-07) from "State Key Laboratory of Genetic Resources and Evolution", Scientific Research Fund of Yunnan Provincial Department of Education (2017ZDX076, 2019 J0646).

\section{Availability of data and materials}

Sequence data from this study were deposited in the GenBank Sequence Read Archive under accession SRA BioProject PRJNA600852.

\section{Declarations}

\section{Ethics approval and consent to participate}

All study procedures involving human subjects were approved by the Medical Ethics Board of the First People's Hospital of Yunnan Province, China. Written and verbal informed consent was obtained from all participants. All methods were performed in accordance with the relevant guidelines and regulations by including a statement.

\section{Consent for publication}

Not applicable.

\section{Competing interests}

There are no conflicts to interest.

\begin{abstract}
Author details
${ }^{1}$ Computational Biology and Medical Ecology Lab, State Key Laboratory of Genetic Resources and Evolution, Kunming Institute of Zoology, Chinese Academy of Sciences, Kunming, China. ${ }^{2}$ Kunming College of Life Sciences, University of Chinese Academy of Sciences, Kunming, China. ${ }^{3}$ Department of Gastroenterology, The First Affiliated Hospital of Kunming Medical University, Yunnan Institute of Digestive Disease, Kunming, Yunnan, China. ${ }^{4}$ Faculty of Science, Kunming University of Science and Technology, Kunming, China. ${ }^{5}$ College of Mathematics, Honghe University, Mengzi, Yunnan Province, China. ${ }^{6}$ Department of General Surgery, The First Affiliated Hospital of Kunming Medical University, Yunnan Institute of Digestive Disease, Kunming, Yunnan, China. ${ }^{7}$ Institute of Fundamental and Frontier Sciences, University of Electronic Science and Technology of China, Chengdu, China. ${ }^{8}$ Center for
\end{abstract}

Excellence in Animal Evolution and Genetics, Chinese Academy of Sciences, Kunming, China.

Received: 3 November 2020 Accepted: 5 April 2021

Published online: 04 May 2021

\section{References}

1. Ananthakrishnan AN. Environmental risk factors for inflammatory bowel diseases: a review. Dig Dis Sci. 2015;60(2):290-8. https://doi.org/10.1007/s1 0620-014-3350-9.

2. Armstrong $H$, Alipour $M$, Valcheva $R$, Bording-Jorgensen $M$, Jovel J, Zaidi $D$, et al. Host immunoglobulin $\mathrm{G}$ selectively identifies pathobionts in pediatric inflammatory bowel diseases. Microbiome. 2019;7(1):1. https://doi.org/10.11 86/s40168-018-0604-3.

3. Bader GD, Hogue CW. An automated method for finding molecular complexes in large protein interaction networks. Bmc Bioinformatics. 2003; 4(1):2-0. https://doi.org/10.1186/1471-2105-4-2.

4. Benckert J, Schmolka N, Kreschel C, Zoller MJ, Sturm A, Wiedenmann B, et al. The majority of intestinal $\lg A+$ and $\lg G+$ plasmablasts in the human gut are antigen-specific. J Clin Investig. 2011;121(5):1946-55. https://doi. org/10.1172/JCl44447.

5. Blakeley-Ruiz JA, Erickson AR, Cantarel BL, Xiong W, Adams R, Jansson JK, et al. Metaproteomics reveals persistent and phylum-redundant metabolic functional stability in adult human gut microbiomes of Crohn's remission patients despite temporal variations in microbial taxa, genomes, and proteomes. Microbiome. 2019;7(1):18. https://doi.org/10.1186/s40168-0190631-8.

6. Borgatti SP, Everett MG. Models of core/periphery structures. Soc Networks. 1999;21(4):375-95. https://doi.org/10.1016/S0378-8733(99)00019-2.

7. Bibiloni R, Mangold M, Madsen KL, Fedorak RN, Tannock GW. The bacteriology of biopsies differs between newly diagnosed, untreated, Crohn's disease and ulcerative colitis patients. J Med Microbiol. 2006;55(8): 1141-9. https://doi.org/10.1099/jmm.0.46498-0.

8. Chao A, Chiu CH, Hsieh TC. Proposing a resolution to debates on diversity partitioning. Ecology. 2012;93(9):2037-51. https://doi.org/10.1890/11-1817.1.

9. Chao A, Chiu CH, Jost L. Unifying species diversity, phylogenetic diversity, functional diversity, and related similarity and differentiation measures through Hill numbers. Annu Rev Ecol Evol Syst. 2014;45(1):297-324. https:// doi.org/10.1146/annurev-ecolsys-120213-091540.

10. Cohen J. Statistical Power Analysis for the Behavioral Sciences (2nd). Hillsdale, NJ: Lawrence Erlbaum Associates; 1988.

11. Cohen LJ, Cho JH, Gevers D, Chu H. Genetic factors and the intestinal microbiome guide development of microbe-based therapies for inflammatory bowel diseases. Gastroenterology. 2019;156(8):2174-89. https://doi.org/10.1053/j.gastro.2019.03.017.

12. Csardi G, Nepusz T. The iGraph software package for complex network research. Inter J Complex Syst. 2006;1695(1). https://doi.org/10.1186/1471-21 05-12-455.

13. Csermely P, London A, Wu LY, Uzzi B. Structure and dynamics of core/ periphery networks. J Complex Netw. 2013;1(2):93-123. https://doi.org/10.1 093/comnet/cnt016.

14. Edgar RC. UPARSE: highly accurate OTU sequences from microbial amplicon reads. Nat Methods. 2013;10(10):996-8. https://doi.org/10.1038/NMETH.2604.

15. Forbes JD, Van Domselaar G, Bernstein CN. Microbiome survey of the inflamed and noninflamed gut at different compartments within the gastrointestinal tract of inflammatory bowel disease patients. Inflamm Bowel Dis. 2016;22(4):817-25. https://doi.org/10.1097/MIB. 0000000000000684

16. Franzosa EA, Sirota-Madi A, Avila-Pacheco J, Fornelos N, Haiser HJ, Reinker S, et al. Gut microbiome structure and metabolic activity in inflammatory bowel disease. Nat Microbiol. 2019;4(2):293-305. https://doi.org/10.1038/s41 564-018-0306-4.

17. Göker M, Gronow S, Zeytun A, Nolan M, Lucas S, Lapidus A, et al. Complete genome sequence of Odoribacter splanchnicus type strain (1651/6). Stand Genomic Sci. 2011;4(2):200-9. https://doi.org/10.4056/ sigs. 1714269 .

18. Halfvarson J, Brislawn CJ, Lamendella R, Vázquez-Baeza Y, Walters WA, Bramer LM, et al. Dynamics of the human gut microbiome in inflammatory bowel disease. Nat Microbiol. 2017;24(6):489-96. https://doi.org/10.1038/ nmicrobiol.2017.4. 
19. Hall AB, Yassour M, Sauk J, Garner A, Jiang X, Arthur T, et al. A novel Ruminococcus gnavus clade enriched in inflammatory bowel disease patients. Genome Med. 2017; 9(1):103. https://doi.org/10.1186/s13073-017-0490-5.

20. Hansen R, Russell RK, Reiff C, Louis P, Mclntosh F, Berry SH, et al. Microbiota of de-novo pediatric IBD: increased Faecalibacterium prausnitzii and reduced bacterial diversity in Crohn's but not in ulcerative colitis. Am J Gastroenterol. 2012;107(12):1913-22. https://doi.org/10.1038/ajg.2012.335.

21. Hanson CA, Fuhrman JA, Horner-Devine MC, Martiny JB. Beyond biogeographic patterns: processes shaping the microbial landscape. Nat Rev Microbiol. 2012;10(7):497-506. https://doi.org/10.1038/nrmicro2795.

22. Heinken A, Ravcheev DA, Baldini F, Heirendt L, Fleming RMT, Thiele I. Systematic assessment of secondary bile acid metabolism in gut microbes reveals distinct metabolic capabilities in inflammatory bowel disease. Microbiome. 2019;7(1):75. https://doi.org/10.1186/s40168-019-0689-3.

23. Henke MT, Kenny DJ, Cassilly CD, Vlamakis H, Xavier RJ, Clardy J. Ruminococcus gnavus, a member of the human gut microbiome associated with Crohn's disease, produces an inflammatory polysaccharide. PNAS. 2019; 116(26):12672-7. https://doi.org/10.1073/pnas.1904099116.

24. Hill MO. Diversity and evenness: a unifying notation and its consequences. Ecology. 1973;54(2):427-32. https://doi.org/10.2307/1934352.

25. Ishikawa D, Sasaki T, Osada T, Kuwahara-Arai K, Haga K, Shibuya T, et al. Changes in intestinal microbiota following combination therapy with fecal microbial transplantation and antibiotics for ulcerative colitis. Inflamm Bowel Dis. 2017;23(1):116-25. https://doi.org/10.1097/MIB 0000000000000975 .

26. Ishikawa D, Sasaki T, Takahashi M, Kuwahara-Arai K, Haga K, Ito S, et al. The microbial composition of Bacteroidetes species in ulcerative colitis is effectively improved by combination therapy with fecal microbiota transplantation and antibiotics. Inflamm Bowel Dis. 2018;24(12):2590-8. https://doi.org/10.1093/ibd/izy266.

27. Jost L. Partitioning diversity into independent alpha and beta components. Ecology. 2007:88(10):2427-39. https://doi.org/10.1890/06-1736.1.

28. Khor B, Gardet A, Xavier RJ. Genetics and pathogenesis of inflammatory bowel disease. Nature. 2011;474(7351):307-17. https://doi.org/10.1038/na ture10209.

29. Kiu R, Caim S, Alcon-Giner C, Belteki G, Clarke P, Pickard D, et al. Preterm infant-associated Clostridium tertium, Clostridium cadaveris, and Clostridium paraputrificum strains: genomic and evolutionary insights. Genome Biol Evol. 2017;9(10):2707-14. https://doi.org/10.1093/gbe/evx210.

30. Knights D, Silverberg MS, Weersma RK, Gevers D, Dijkstra G, Huang H, et al. Complex host genetics influence the microbiome in inflammatory bowel disease. Genome Med. 2014;6(12):107. https://doi.org/10.1186/s13073-014-0107-1

31. Kostic AD, Xavier RJ, Gevers D. The microbiome in inflammatory bowel disease: current status and the future ahead. Gastroenterology. 2014;146(6): 1489-99. https://doi.org/10.1053/j.gastro.2014.02.009

32. Kump PK, Gröchenig HP, Lackner S, Trajanoski S, Reicht G, Hoffmann KM, et al. Alteration of intestinal dysbiosis by fecal microbiota transplantation does not induce remission in patients with chronic active ulcerative colitis Inflamm Bowel Dis. 2013;19(10):2155-65. https://doi.org/10.1097/MIB.0b013 e31829ea325.

33. Lee D, Albenberg L, Compher C, Baldassano R, Piccoli D, Lewis JD, et al. Diet in the pathogenesis and treatment of inflammatory bowel diseases. Gastroenterology. 2015;148(6):1087-106. https://doi.org/10.1053/j.gastro.

34. Li W, Ma ZS. Diversity scaling of human vaginal microbial communities. Zool Res. 2019:40(6):587-94. https://doi.org/10.24272/j.issn.2095-8137.2019.068.

35. Lloyd-Price J, Arze C, Ananthakrishnan AN, et al. Multi-omics of the gut microbial ecosystem in inflammatory bowel diseases. Nature. 2019; 569(7758):655-62. https://doi.org/10.1038/s41586-019-1237-9.

36. Ma ZS. The P/N (positive-to-negative links) ratio in complex networks-a promising in Silico biomarker for detecting changes occurring in the human microbiome. Microb Ecol. 2018;75(4):1063-73. https://doi.org/10.1 007/s00248-017-1079-7.

37. Ma ZS, Ellison AM. Dominance network analysis provides a new framework for studying the diversity-stability relationship. Ecol Monogr. 2019;89(2): e01358. https://doi.org/10.1002/ecm.1358.

38. Ma ZS, Li L, Gotelli NJ. Diversity-disease relationships and shared species analyses for human microbiome-associated diseases. ISME J. 2019;13(8): 1911-9. https://doi.org/10.1038/s41396-019-0395-y.

39. Ma ZS, Li W. How and why men and women differ in their microbiomes: medical ecology and network analyses of the Microgenderome. Adv Sci. 2019;6(23):1902054. https://doi.org/10.1002/advs.201902054.
40. Macpherson A, Khoo UY, Forgacs I, Philpott-Howard J, Bjarnason I. Mucosal antibodies in inflammatory bowel disease are directed against intestinal bacteria. Gut. 1996;38(3):365-75. https://doi.org/10.1136/gut.38.3.365.

41. Miyoshi J, Chang EB. The gut microbiota and inflammatory bowel diseases. Transl Res. 2017;179:38-48. https://doi.org/10.1016/j.trsl.2016.06.002.

42. Morgan XC, Tickle TL, Sokol H, Gevers D, Devaney KL, Ward DV, et al. Dysfunction of the intestinal microbiome in inflammatory bowel disease and treatment. Genome Biol. 2012;13(9):R79. https://doi.org/10.1186/gb-2 012-13-9-r79.

43. Nemoto H, Kataoka K, Ishikawa H, Ikata K, Arimochi H, Iwasaki T, et al. Reduced diversity and imbalance of fecal microbiota in patients with ulcerative colitis. Dig Dis Sci. 2012;57(11):2955-64. https://doi.org/10.1007/s1 0620-012-2236-y.

44. Ni J, Wu GD, Albenberg L, Tomov VT. Gut microbiota and IBD: causation or correlation? Nat Rev Gastroenterol Hepatol. 2017;14(10):573-84. https://doi. org/10.1038/nrgastro.2017.88.

45. Nishida A, Inoue $R$, Inatomi O, Bamba S, Naito $Y$, Andoh A. Gut microbiota in the pathogenesis of inflammatory bowel disease. Clin J Gastroenterol. 2018;11(1):1-10. https://doi.org/10.1007/s12328-017-0813-5.

46. Nishino K, Nishida A, Inoue R, Kawada Y, Ohno M, Sakai S, et al. Analysis of endoscopic brush samples identified mucosa-associated dysbiosis in inflammatory bowel disease. J Gastroenterol. 2018;53(1):95-106. https://doi. org/10.1007/s00535-017-1384-4

47. Pike BL, Paden KA, Alcala AN, Jaep KM, Gormley RP, Maue AC, et al. Immunological biomarkers in Postinfectious irritable bowel syndrome. J Travel Med. 2015;22(4):242-50. https://doi.org/10.1111/jtm.12218.

48. Rajca S, Grondin V, Louis E, Vernier-Massouille G, Grimaud JC, Bouhnik Y, et al. Alterations in the intestinal microbiome (dysbiosis) as a predictor of relapse after infliximab withdrawal in Crohn's disease. Inflamm Bowel Dis. 2014:20(6):978-86. https://doi.org/10.1097/MIB.0000000000000036.

49. Ray $P$, Das A, Singh $K$, Bhansali A, Yadav TD. Clostridium tertium in necrotizing fasciitis and gangrene. Emerg Infect Dis. 2003;9(10):1347-8. https://doi.org/10.3201/eid0910.030287.

50. Salvador F, Porte L, Durán L, Marcotti A, Pérez J, Thompson L, et al. Breakthrough bacteremia due to Clostridium tertium in a patient with neutropenic fever, and identification by MALDI-TOF mass spectrometry. Int Infect Dis. 2013;17(11):e1062-3. https://doi.org/10.1016/j.ijid.2013.03.005.

51. Sartor RB, Wu GD. Roles for Intestinal Bacteria, Viruses, and Fungi in Pathogenesis of Inflammatory Bowel Diseases and Therapeutic Approaches. Gastroenterology. 2017;152(2):327-339.e4. https://doi.org/1 0.1053/j.gastro.

52. Shah R, Cope JL, Nagy-Szakal D, Dowd S, Versalovic J, Hollister EB, et al. Composition and function of the pediatric colonic mucosal microbiome in untreated patients with ulcerative colitis. Gut Microbes. 2016;7(5):384-96. https://doi.org/10.1080/19490976.2016.1190073.

53. Shah S, Hankenson J, Pabbathi S, Greene J, Nanjappa S. Clostridium tertium in neutropenic patients: case series at a cancer institute. Int J Infect Dis. 2016:51(C):44-6. https://doi.org/10.1016/j.ijid.2016.08.013.

54. Shannon P, Markiel A, Ozier O, Baliga NS, Wang JT, Ramage D, et al. Cytoscape: a software environment for integrated models of biomolecular interaction networks. Genome Res. 2003;13(11):2498-504. https://doi.org/1 $0.1101 /$ gr. 1239303

55. Sheehan D, Moran C, Shanahan F. The microbiota in inflammatory bowel disease. J Gastroenterol. 2015;50(5):495-507. https://doi.org/10.1007/s00535015-1064-1.

56. Sokol H. Probiotics and antibiotics in IBD. Dig Dis. 2014;32(Suppl 1):10-7. https://doi.org/10.1159/000367820.

57. Steensma EA, Ertl CW, Burke LH. Clostridium tertium isolated from a necrotizing soft tissue infection in a diabetic but otherwise nonimmunocompromised patient. J Am Col Certif Wound Spec. 2011;3(2): 42-4. https://doi.org/10.1016/j.jcws.2011.09.002.

58. Sutton SS, Jumper M, Shah A, Edun B. Clostridium tertium peritonitis and concurrent bacteremia in a patient with a history of alcoholic cirrhosis. J Investig Med High Impact Case Rep. 2017:5(3):2324709617731457. https:// doi.org/10.1177/2324709617731457.

59. Takahashi K, Nishida A, Fujimoto T, Fujii M, Shioya M, Imaeda H, et al. Reduced abundance of butyrate-producing Bacteria species in the fecal microbial Community in Crohn's disease. Digestion. 2016;93(1):59-65. https://doi.org/10.1159/000441768.

60. Vanderhofstadt $M$, André $M$, Lonchay $C$, Levecque $P$, Holemans $X$, Canon JL, et al. Clostridium tertium bacteremia: contamination or true pathogen? A 
report of two cases and a review of the literature. Int J Infect Dis. 2010; 14(Suppl 3):e335-7. https://doi.org/10.1016/j.ijid.2010.03.004.

61. Vellend M. Conceptual synthesis in community ecology. Q Rev Biol. 2010; 85(2):183-206. https://doi.org/10.1086/652373.

62. Wazir M, Jain AG, Nadeem M, Ur Rahman A, Everett G. Clostridium tertium bacteremia in a non-neutropenic patient with liver cirrhosis. Cureus. 2019; 11(4):e4432. https://doi.org/10.7759/cureus.4432.

63. Wei Y, Zhu W, Gong J, Guo D, Gu L, Li N, et al. Fecal microbiota transplantation improves the quality of life in patients with inflammatory bowel disease. Gastroenterol Res Pract. 2015;517597:1-5. https://doi.org/1 $0.1155 / 2015 / 517597$.

64. Weingarden AR, Vaughn BP. Intestinal microbiota, fecal microbiota transplantation, and inflammatory bowel disease. Gut Microbes. 2017;8(3): 238-52. https://doi.org/10.1080/19490976.

65. Willing BP, Dicksved J, Halfvarson J, Andersson AF, Lucio M, Zheng Z, et al. A pyrosequencing study in twins shows that gastrointestinal microbial profiles vary with inflammatory bowel disease phenotypes. Gastroenterology. 2010;139(6):1844-1854.e1. https://doi.org/10.1053/j.ga stro.2010.08.049

\section{Publisher's Note}

Springer Nature remains neutral with regard to jurisdictional claims in published maps and institutional affiliations.

Ready to submit your research? Choose BMC and benefit from:

- fast, convenient online submission

- thorough peer review by experienced researchers in your field

- rapid publication on acceptance

- support for research data, including large and complex data types

- gold Open Access which fosters wider collaboration and increased citations

- maximum visibility for your research: over $100 \mathrm{M}$ website views per year

At BMC, research is always in progress.

Learn more biomedcentral.com/submissions 